\title{
Thanks to CLD for Small Favors: Reduced CVD Risk in Patients Awaiting Liver Transplantation
}

\author{
Hersh Shroff ${ }^{1} \cdot$ Mary E. Rinella ${ }^{1}$ \\ Published online: 29 May 2020 \\ (c) Springer Science+Business Media, LLC, part of Springer Nature 2020
}

Over the past two decades, there has been a shift in the prevailing etiologies of chronic liver disease (CLD), both in the USA and worldwide [1]. Since the advent of direct acting antivirals (DAAs), the burden of hepatitis $C$ virus (HCV)-related CLD has steadily decreased, whereas that of nonalcoholic fatty liver disease (NAFLD) has increased, attributable largely to higher global rates of obesity and diabetes. As a result, nonalcoholic steatohepatitis (NASH) and alcoholic liver disease (ALD) have now surpassed HCV as the leading indications for liver transplant (LT) evaluation in the USA [2].

Cardiovascular disease (CVD) is increasingly recognized as an important comorbidity in patients with liver disease and NASH in particular [3]. As CLD progresses to advanced fibrosis and end-stage liver disease (ESLD), the rate of liverrelated events increases 40-fold [4], with CVD incidence remaining elevated [5]. Since numerous factors impact CV risk in patients with NASH, such factors are likely to evolve over the course of liver disease progression. In addition to the competing risk of increased liver-related death, physiologic changes related to portal hypertension or impaired hepatic synthetic function may alter CV risk (e.g., reduction in systemic arterial pressure, alteration of cardiac structure and impairment of cardiac function, alterations in coagulation balance, and changes in hepatic lipid metabolism).

In the current issue of Digestive Diseases and Sciences, Patel et al. [6] present data from a cohort of 682 patients with ESLD of several etiologies undergoing LT evaluation, comparing the rate of $\mathrm{CV}$ events to the rate of non-cardiac death and/or ultimate LT. Here, they show that in their cohort of patients being evaluated for LT, $3.4 \%$ had a CV

Mary E. Rinella

m-rinella@ northwestern.edu

1 Division of Gastroenterology and Hepatology, Department of Medicine, Northwestern University Feinberg School of Medicine, 676 N. St Clair St, Suite 14-012, Chicago, IL 60611, USA event (symptomatic arrhythmia, acute coronary syndrome [ACS], new-onset heart failure, stroke, or cardiac death), including a $0.9 \%$ rate of ACS, over a median follow-up of 585 days. In a competing risk model, the $3.4 \%$ rate of CVD events was significantly lower than the $73.6 \%$ likelihood of undergoing LT or experiencing non-cardiac death. When the analysis was restricted to the rate of non-cardiac death only in patients who did not ultimately undergo LT (i.e., were declined at time of evaluation), the lower rate of $\mathrm{CV}$ events remained significant. Not surprisingly, the presence of CAD at the time of LT evaluation (determined by coronary angiography, performed in all patients over the age of 50 or in those with CV risk factors), was significantly associated with the risk of $\mathrm{CV}$ events.

In order to elucidate potential mechanisms to explain the observed lower rate of $\mathrm{CV}$ events in patients with cirrhosis listed for LT, the authors examined differences in biomarkers of $\mathrm{CV}$ risk, including lipoprotein sub-fractions, between matched cohorts of NASH patients with and without cirrhosis. While the authors found no differences between the groups in markers of chronic inflammation (fibrinogen, high sensitivity C-reactive protein [hs-CRP], and lipoprotein-associated phospholipase A2 [Lp-PLA2]), those with cirrhosis surprisingly had a significantly less atherogenic serum lipid profile. The authors convincingly demonstrate that patients with NASH cirrhosis, in addition to having lower serum LDL and triglycerides compared with those without cirrhosis, also have significant differences in lipoprotein sub-fractions: decreased small dense low-density lipoprotein cholesterol (sdLDL)-C (18.9 \pm 9.1 vs. $37.2 \pm 16.1 \mathrm{mg} / \mathrm{dL}$ ), decreased very-low-density lipoprotein $(\mathrm{VLDL})(2.1 \pm 1.2$ vs. $4.3 \pm 0.5 \mathrm{nmol} / \mathrm{L})$, and increased high-density lipoprotein (HDL)-2 (21.04 \pm 12.9 vs. $11.6 \pm 5.2 \mathrm{mg} / \mathrm{dL})$. Reductions in sdLDL coupled with the increase in HDL-2 should reduce atherosclerotic risk. Interestingly, patients with NASH cirrhosis also had increased serum adiponectin levels despite no significant difference in insulin levels or hemoglobin A1C. The 


\section{Drivers of mortality in the peri-transplant period}

Pre-liver transplant

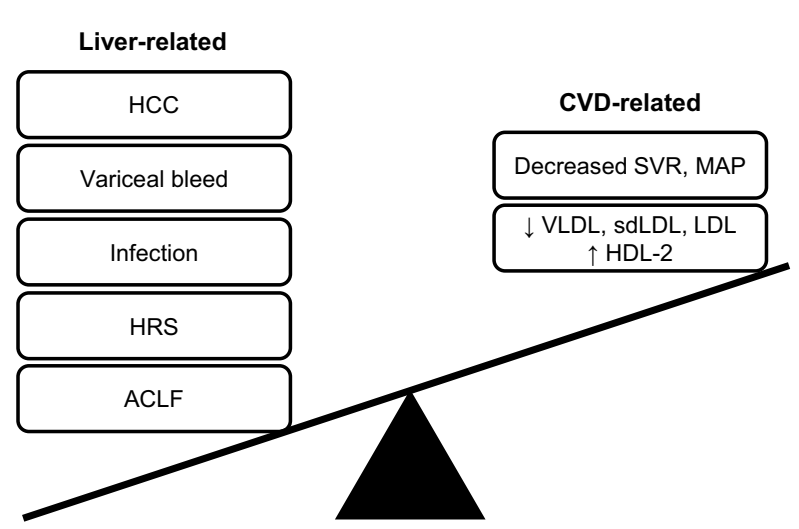

Fig. 1 Drivers of mortality in the peri-transplant period. ACLF acuteon-chronic liver failure, $B P$ blood pressure, $H C C$ hepatocellular carcinoma, $H D L-2$ high-density lipoprotein-2, HRS hepatorenal syn-

authors hypothesize that this may be explained by "hepatogenous" diabetes, resulting from portosystemic shunting, which is plausible but requires further investigation.

Prior cross-sectional studies have demonstrated that advanced fibrosis (determined both by clinical prediction rules such as the FIB-4 score and by histology) is an independent predictor of subclinical atherosclerosis [7-9]. Thus, the mechanistic link proposed in this study would be bolstered by future studies identifying improvements in the severity of atherosclerotic plaques, reflective of the reduced atherogenicity of serum lipid profiles in those with cirrhosis followed over time. The major challenge with this is that patients with ESLD undergoing LT evaluation have poor long-term survival and may lack the duration of follow-up necessary to demonstrate meaningful changes in atherosclerotic burden.

A population consisting only of patients referred for LT evaluation is likely biased toward a more favorable cardiac risk profile, limiting the generalizability of the findings to the broader population of individuals with cirrhosis. In a prospective, longitudinal study of 1702 adults in the NASH Clinical Research Network (CRN) database, CVD incidence was higher in individuals with NASH and advanced fibrosis than in those at earlier disease stages [5]. Furthermore, in a phase 3 trial of patients with advanced fibrosis due to NASH, those with cirrhosis were significantly more likely to have a major adverse cardiac event than those with stage 3 fibrosis. Thus, the lipoprotein alterations observed in the pre-LT NASH cirrhosis cohort presented by Patel et al. may be insufficient to mitigate CVD risk in the broader cirrhosis population. In this regard,
Post-liver transplant

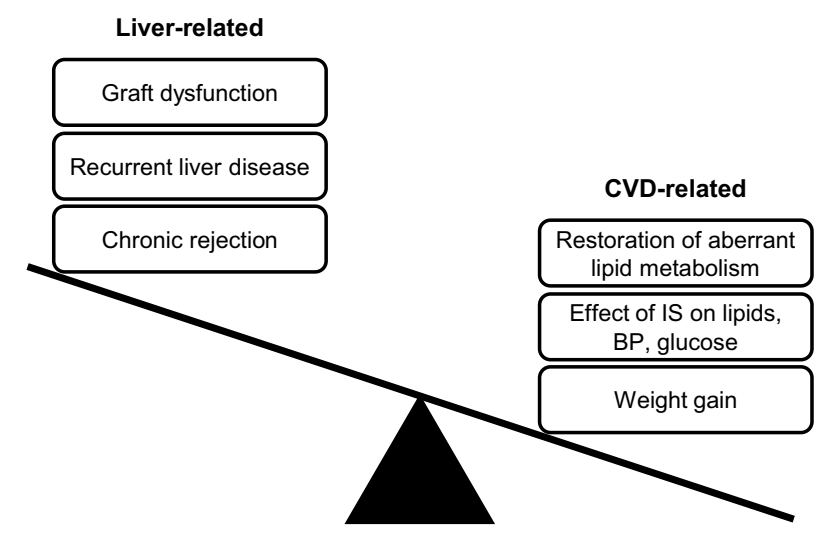

drome, $I S$ immunosuppressives, $L D L$ low-density lipoprotein, $M A P$ mean arterial pressure, $s d L D L$ small dense low-density lipoprotein, $S V R$ systemic vascular resistance, $V L D L$ very-low-density lipoprotein

future larger studies with less restricted populations will be important to further confirm the current findings.

Given the limited survival in individuals with ESLD awaiting transplant, it is perhaps more relevant to focus on $\mathrm{CV}$ risk in these patients after they undergo liver transplantation, when the competing risk of hepatic dysfunction is substantially diminished. The majority of individuals with ESLD who do not undergo LT are far more likely to experience a liver-related event than to survive to experience any clinically meaningful change in CV events. Nevertheless, in those who ultimately do undergo LT, particularly in those transplanted for NASH cirrhosis, adverse CV outcomes are increased (Fig. 1) [10, 11]. This is explained by numerous factors, including detrimental metabolic effects of immunosuppressant (IS) medications, re-establishment of weight gain, and either the return of aberrant lipid metabolism with restoration of normal hepatic function or its accentuation by IS medications [12]. An improved understanding of the evolution of lipid metabolic changes occurring in cirrhosis, as identified in this study, compared with those occurring after LT would further improve the understanding of CV risk in the peri-transplant setting.

The present study by Patel et al. adds valuable information to the understanding of the contribution of the liver to regulating mediators of atherosclerosis, generating important questions regarding cardiovascular risk in advanced liver disease. For one, the study confirms that despite the presence of underlying $\mathrm{CV}$ risk factors such as $\mathrm{CAD}$, liverrelated morbidity and mortality substantially outweigh $\mathrm{CV}$ events in this subpopulation of patients with ESLD. Though the severity and frequency of complications associated with advanced liver disease is likely a major driver of this 
finding, we agree that it is nevertheless important to consider whether additional pathophysiologic mechanisms in cirrhosis may impact overall $\mathrm{CV}$ risk. To that end, a reduction in atherogenicity of serum lipid profiles and improvements in serum adiponectin occurring in individuals with NASH cirrhosis may provide relative cardioprotection. Future studies should focus on corroborating the mechanisms of changing $\mathrm{CV}$ risk in non-NASH etiologies of CLD and on further elucidating the long-term clinical impact of these changes.

\section{References}

1. Kim D, Li AA, Gadiparthi C, et al. Changing trends in etiologybased annual mortality from chronic liver disease, from 2007 through 2016. Gastroenterology. 2018;155(4):1154-1163. https ://doi.org/10.1053/j.gastro.2018.07.008. e3.

2. Wong RJ, Aguilar M, Cheung R, et al. Nonalcoholic steatohepatitis is the second leading etiology of liver disease among adults awaiting liver transplantation in the United States. Gastroenterology. 2015;148(3):547-555. https://doi.org/10.1053/j.gastr o.2014.11.039

3. Kim D, Adejumo AC, Yoo ER, et al. Trends in mortality from extrahepatic complications in patients with chronic liver disease, from 2007 through 2017. Gastroenterology. 2019;157(4):10551066. https://doi.org/10.1053/j.gastro.2019.06.026. e11.

4. Dulai PS, Singh S, Patel J, et al. Increased risk of mortality by fibrosis stage in nonalcoholic fatty liver disease: systematic review and meta-analysis. Hepatology. 2017;65(5):1557-1565. https:// doi.org/10.1002/hep.29085

5. Sanyal AJ, Van Natta ML, Lazo M, et al. A prospective longitudinal study of clinical outcomes in adults with nonalcoholic fatty liver disease. Hepatology. 2019;70(Suppl 1):717A-718A.
6. Patel S, Siddiqui MB, Chandrakumaran A, Rodriguez VA, Faridnia M, Hernandez Roman J et al. Progression to cirrhosis leads to improvement in atherogenic milieu. Dig Dis Sci. (Epub ahead of print). https://doi.org/10.1007/s10620-020-06196-4.

7. Arai T, Atsukawa M, Tsubota A, et al. Factors influencing subclinical atherosclerosis in patients with biopsy-proven nonalcoholic fatty liver disease. PLoS One.. 2019;14(11):e0224184. https://doi. org/10.1371/journal.pone.0224184.

8. Song DS, Chang UI, Kang SG, Song SW, Yang JM. Noninvasive serum fibrosis markers are associated with coronary artery calcification in patients with nonalcoholic fatty liver disease. Gut Liver. 2019;13(6):658-668. https://doi.org/10.5009/gnl18439.

9. Sunbul M, Agirbasli M, Durmus E, et al. Arterial stiffness in patients with non-alcoholic fatty liver disease is related to fibrosis stage and epicardial adipose tissue thickness. Atherosclerosis. 2014;237(2):490-493. https://doi.org/10.1016/j.atherosclerosis .2014.10.004.

10. Vanwagner LB, Bhave M, Te HS, Feinglass J, Alvarez L, Rinella ME. Patients transplanted for nonalcoholic steatohepatitis are at increased risk for postoperative cardiovascular events. Hepatology. 2012;56(5):1741-1750. https://doi.org/10.1002/hep.25855.

11. VanWagner LB, Serper M, Kang R, et al. Factors associated with major adverse cardiovascular events after liver transplantation among a national sample. Am J Transplant. 2016;16(9):26842694. https://doi.org/10.1111/ajt.13779.

12. Izzy M, VanWagner LB, Lee SS, Altieri M, Angirekula M, Watt KD. Understanding and managing cardiovascular outcomes in liver transplant recipients. Curr Opin Organ Transplant. 2019;24(2):148-155. https://doi.org/10.1097/MOT.0000000000 000614 .

Publisher's Note Springer Nature remains neutral with regard to jurisdictional claims in published maps and institutional affiliations. 